FEDSM2002-31010

\title{
NUMERICAL INVESTIGATION OF BUBBLE CLOUD DYNAMICS IN SHOCK WAVE LITHOTRIPSY
}

\author{
Michel Tanguay \\ Tim Colonius \\ Department of Mechanical Engineering \\ California Institute of Technology Pasadena, California 91125 \\ Email: michel@caltech.edu
}

\begin{abstract}
To provide greater understanding of some of the phenomena in Extracorporeal Shock Wave Lithotripsy (ESWL), we implemented a two-phase continuum model for cavitating flow and applied it to the simulation of bubble cloud dynamics in an electrohydraulic lithotripter. Through the combination of a WENO shock capturing scheme, curvilinear coordinates system and ensemble averaged mixture model, we computed the evolution of the lithotripsy shock wave and the concomitant cavitation field. In this paper, we present the results for three different configurations: a single-pulse lithotripter (free field), a single-pulse lithotripter with rigid artificial kidney stone at the focal point, and a dual-pulse lithotripter. Qualitative and quantitative comparisons of the numerical results to experimental observations are also included.
\end{abstract}

\section{INTRODUCTION}

Extracorporeal shock wave lithotripsy (ESWL) continues to be one of the most popular types of intervention in the treatment of kidney stone disease. Through the firing of focused shock waves, stones are reduced to fragments sufficiently small for natural elimination. Like any other medical procedure, ESWL can be optimized by reducing side effects while maximizing its potency. The impact of thousands of shock waves (a typical treatment may consists of 1000-3000 shocks in a period of 20-30 minutes) on a kidney includes subcapsular hematomas (bleeding inside the kidney) as well as morphological changes (Coleman \& Saunders (1993), Willis et al. (1999)). As for stone comminu- tion, the shock wave generates internal stresses as it hits the stone which may be sufficient to cause failure (spalling) but also damages the stone indirectly via cavitation (erosion) that is produced by the tensile component of the shock.

Empirical observations of the cavitation field are very limited and it is extremely difficult to ascertain how to optimize the ESWL pulse for enhanced cavitation and stone comminution. That is precisely where we believe that a numerical lithotripter model can make an important contribution and provide information that would otherwise be unattainable. Previous computational modeling in this field have consisted for the most part in the numerical integration of bubble size (using Rayleigh-Plesset, Gilmore or similar model) using an experimentally measured pressure trace as the forcing term Bailey et al. (1999) and Cleveland et al. (2000)). The results obtained following this decoupled approach are then compared to the output signal of passive cavitation detectors. Other studies such as Averkiou \& Cleveland (1999), used non-linear acoustics to predict the pressure at the focal point. However, based on the work of Lokhandwalla \& Sturtevant (2001), it can be shown that the presence of a solid object at the focal point can alter the behavior of bubbles beyond what is predicted by a decoupled approach. Furthermore, the work of Kumar \& Brennen (1993) and Wang (1999) has clearly demonstrated that cavitation can play a significant role in the overall dynamics of the bubbly mixture. In our past work Tanguay \& Colonius (2001), we have presented preliminary results which indicate that the presence of cavitating bubbles play a measurable role in the focusing process of the ESWL wave. 




Figure 1. Diagram of a electro-hydraulic lithotripter.

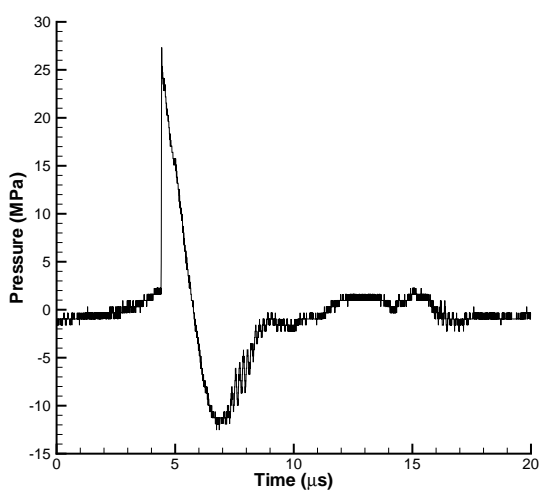

Figure 2. Pressure measured at the focus of an electro-hydraulic lithotripter as a function of time. Courtesy of Michael R. Bailey, Center for Industrial and Medical Ultrasound, Applied Physics Lab, University of Washington, Seattle.

\section{MODELING}

\section{Physical aspects of lithotripsy}

The basic electro-hydraulic lithotripter consists of a spark generator and an ellipsoidal reflector (see figure 1). A spark fired at one of the focal points of the reflector (referred to as $F_{1}$ ) generates an expanding spherical shock wave. Part of this wave is then refocused by the ellipsoidal reflector to the second focal point $\left(F_{2}\right)$. As discussed in Averkiou \& Cleveland (1999), the initial spherical pulse can be approximated by a positive triangular wave.

A typical pressure trace at the $F_{2}$ is shown in figure 2. The tensile portion of the wave form is caused in part by the focusing process and the interaction between the incident wave and the edge of the reflector (edge wave). In its wake, a narrow region of cavitation bubbles can be observed (figure 3 ).

\section{Numerical model}

We consider a continuum bubbly flow model based on the work of Zhang \& Prosperetti (1994a) and (1994b). The governing equations for the average mixture properties are obtained by ensemble averaging over all bubble states and locations. The equations are then simplified in the limit of low void fraction.
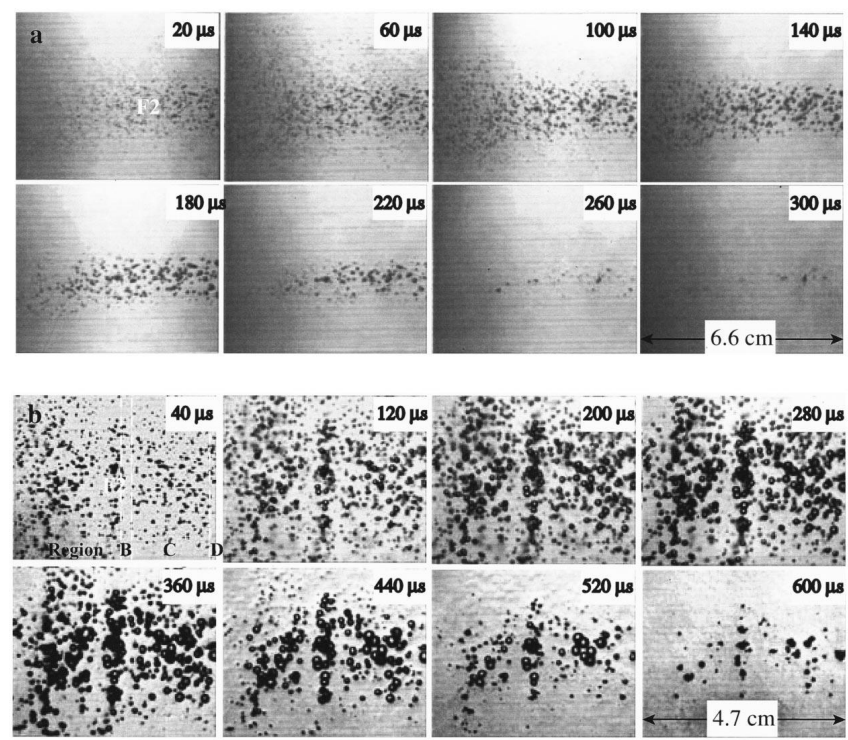

Figure 3. Snapshots of a bubble cloud generated by an electro-hydraulic lithotripter. The first height frames are for the free field case and the last height are for the dual-pulse lithotripter. Courtesy of Dahlia R. Sokolov, Center for Industrial and Medical Ultrasound, Applied Physics Lab, University of Washington, Seattle.

Within this approximation, the equations used in this work are similar to the ones obtained from the analysis of Biesheuvel \& van Wijngaarden (1984). Only a brief discussion of some of the important assumptions of the model will be presented here.

Most of the key assumptions of the model relate to the derivation of the governing equations for the bubble field. The conditions of the gas inside a bubble are assumed to be thoroughly mixed and of uniform temperature which is equivalent to neglecting mass and heat transfer within the gas phase. The low void fraction assumption mentioned above also allows for the omission of direct bubble-bubble interactions, which means that bubbles can only influence each other via the void fraction and mixture pressure. Bubbles are assumed to be small compared to length scales associated with variations in the mixture properties. This allows for the flow field surrounding the bubble to be approximated as incompressible and the far-field pressure to be approximated by the local mixture value.

In the derivation of the ensemble averaged equations, terms involving the average of functions of bubble states appear. For the purpose of this study, we have assume that the average of a function is equal to the function of the average states $(\langle f(R, \dot{R})\rangle \approx f(\langle R\rangle,\langle\dot{R}\rangle))$. This is consistent with the assumption that the probability density field for the bubble states at a particular location in space is a delta function centered at the average states. Work is currently underway to relax this assumption and directly compute the evolution of the probability density 
function.

Some of these approximations are restrictive and we are currently working to extend the capabilities of the present approach. An extension to the bubble model which will include a correction due to the interaction between large pressure gradients (namely the shock wave in this case) and bubble dynamics is currently under development. In addition, the approach developed in Preston et al. (2002) to approximate the bubble damping due to thermal effect will be introduced in the near future.

\section{NUMERICAL IMPLEMENTATION}

The interaction between the incident wave and the reflector is an essential component for a realistic numerical model of a lithotripter. In order to accurately capture the surface, a prolate spheroidal coordinates system was implemented over part of the domain (see figure 4).

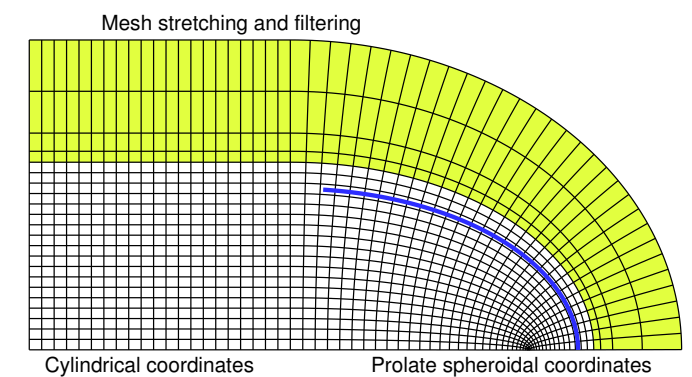

Figure 4. Mesh representation

In order to accurately represent the shock wave, a Weighted Essentially Non-Oscillatory (WENO) fifth-order shock-capturing scheme was implemented. Details regarding the implementation can be found in the work of Liu et al. (1994) and Jiang \& Shu (1996). The time integration was performed using an explicit third-order Total Variation Diminishing (TVD) scheme.

The prolate spheroidal coordinates system has a singularity at the focal point. The CFL stability limit near this singularity is too restrictive for explicit time integration. Because of this, an implicit first-order upwinding time integration scheme was implemented in this region (see figure 5).

The implementation of the radial boundary condition required some special attention. The approach used here follows from the work of Colonius et al. (1993). At the edge of the domain, a non-reflective boundary condition similar to the one presented in Poinsot \& Lele (1992) is used. Because the governing equations are axisymmetric and cannot be formulated in conservative form, the numerical boundary can introduce a nonnegligible spurious reflection. The magnitude of this artifact di-

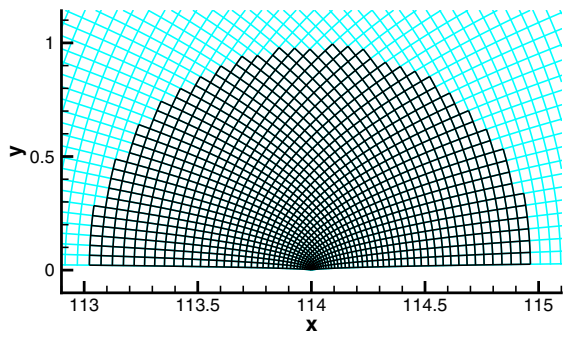

Figure 5. Mesh near the coordinate singularity. The implicit domain is shown in black.

minishes with distance from axis. Hence, the mesh is stretched radially in the outer region of the domain. The solution in the stretched region is continuously filtered in order to prevent the higher frequency modes from begin aliased back into the domain.

The centerline treatment was based on the work of Mohseni $\&$ Colonius (2000). A similar implementation was used on all reflective surfaces (ellipsoidal reflector and artificial stone) as well as for plane of symmetry (for the dual-pulse lithotripter test case).

Since the spark generation cannot be simulated from first principles, an approach based on the work presented by Averkiou \& Cleveland (1999) was used. The expanding shock wave is approximated by triangular wave form of peak pressure of $4 \mathrm{MPa}$ and $6 \mathrm{~mm}$ thickness starting $1 \mathrm{~cm}$ away from $F_{1}$. To prescribe an outgoing positive wave form with a compatible velocity profile (zero velocity in front of the pulse), a constant mass source term was added to the governing equations. This mass flux represents the expansion of the cavity created by the initial vaporization of liquid by the spark. Under real circumstances, this cavity collapses after some time. This effect was neglected at this stage.

The forcing terms for the disperse phase was integrated separately within each time step using a fifth-order Kaps-Rentrop adaptive time marching algorithm.

Although this implementation shares many similarities to that of our earlier work Tanguay \& Colonius (2001), it benefits from several improvements. In the previous implementation, the calculation domain was divided into two parts: the reflector domain (prolate spheroidal coordinates, finite-difference) and the cavitation domain (cylindrical coordinates, ENO shockcapturing). The calculations were first performed in the reflector domain and then the output used in the cavitation domain. Due to the finite-difference implementation, a much smoother initial pulse had to be used. In the current approach, both regions are directly connected and benefit from the WENO scheme.

\section{RESULTS}

Our numerical results for the cavitation cloud produced by a lithotripter are presented here. The first case of interest is the 
simulation of a lithotripter with no obstructions in the field (free field). This case serves as a benchmark test for both the numerical algorithm and the simulation parameters such as initial bubble radius and bubble number density. In the second test case, we introduce an artificial stone (infinite stiffness) at the focal point. The final case presented in this work involves two aligned reflector fired simultaneously at the same focal point. This is equivalent to introducing a flat reflector at the focal point. This case was first introduced by Sokolov et al. (2000) to localize and intensify the cavitation at $F_{2}$.

\section{Free field lithotripter}

This case is the first benchmark for the numerical model and also serves as a baseline comparison for all other cases. The key parameters for the simulation other than the geometry of the reflector are the initial equilibrium bubble size $\left(R_{o}=10 \mu \mathrm{m}\right)$ and the initial bubble number density $\left(\eta=37.5\right.$ bubbles $\left./ \mathrm{m}^{3}\right)$, water properties. The value of the bubble number density is based on estimated experimental values. As for the equilibrium radius, the value cannot be obtained from experiments since the nucleation sites are too small for measurement. Instead, we must guess a value which yields output results compatible with empirical observations. Based on preliminary numerical results, the value of $R_{o}$ was set to $10 \mu \mathrm{m}$. All water properties were set to tabulated values for standard atmospheric pressure and temperature.

One of the outputs frequently measured experimentally is the pressure as function of time at the focal point. A comparison between a typical trace and our present numerical model is shown in figure 6 . The peak positive pressure for the numerical calculation compares well with the experimental value. The negative pressure obtained using the model with and without cavitation present is significantly smaller than the empirical values. The discrepancy can in part be explained by the continuous use of the mass source term at the spark. As mentioned before, the present model does not account for the decay of this term.

A snapshot for the bubble cloud cavitation is presented in figure 7. The thickness of the bubble cloud varies from $10-12 \mathrm{~mm}$ diameter and its length is approximately $70 \mathrm{~mm}$ at its maximum. As seen previously in figure 3 , these values are close to the dimensions of the bubble cloud observed in experiments.

The bubble size at the focus as a function of time can be found in figure 8 . The maximum bubble size for this calculation is approximately $0.85 \mathrm{~mm}$ which is well within the range of measured values. As seen in figure 8 , the time between violent collapse is approximately $320 \mu$ s which corresponds very well with the measurements obtained with passive cavitation detectors. Some snapshots of the pressure and void fraction near the focal point can be found in figures 9 and 10 .

Although the present results agree quite well in some respect with their experimental counterparts, they digresses from observation in that no large after-bounces are usually observed after a

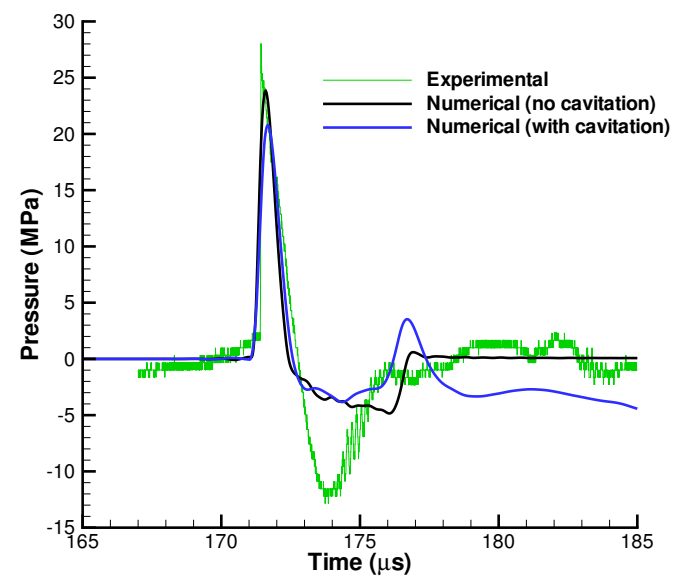

Figure 6. Comparison between results obtained with and without cavitation to experimental measurements.

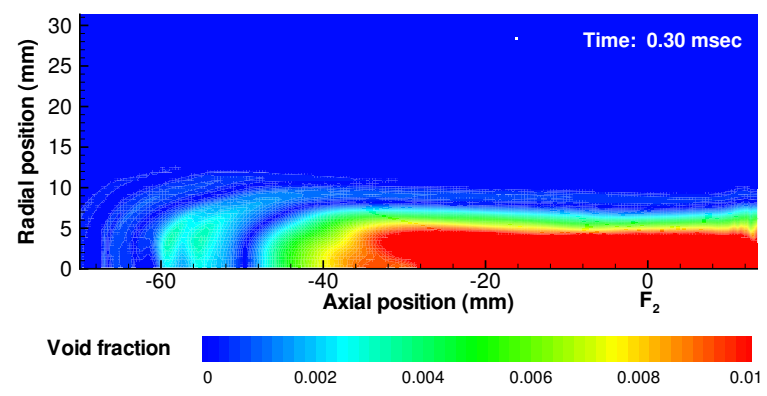

Figure 7. Void fraction contour for free field lithotripter calculations $\left(F_{2}\right.$ is located at zero on the abscissa.)

bubble violent collapse. This limitation in the bubble model is yet to be resolved. Some current work in this area can be found in Matula et al. (2002).

\section{Lithotripsy on artificial stone and dual-pulse lithotripter}

For the second test case, a cylindrical rigid stone was introduced at the focal point. This case is somewhat similar to in vitro studies where artificial stones are used. However, in the experimental setting, the stone has some limited but non-negligible compliance and mobility.

The third case presented here is the dual-pulse lithotripter. This case holds particular importance since it provides a crucial test for the numerical model. In their experimental results, Sokolov et al. (2001) have noted that the cavitation field exhibited a banded structure (see figure 3). Close to the focal point, some regions of the cavitation cloud appear to be devoid of measurable bubble activity. In addition, the bubbles located closest to the focal point were observed to grow nearly twice as much 


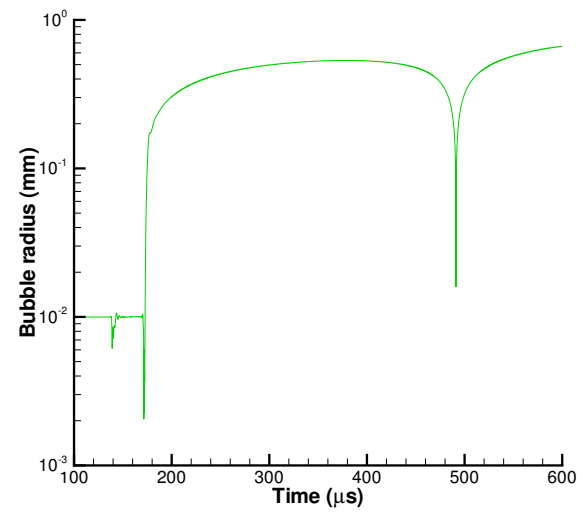

Figure 8. Bubble radius as a function of time at $F_{2}$ for free field lithotripter simulation with cavitation

and last for almost twice as long as bubbles in a single pulse lithotripter (free field). The increased growth was attributed to the superposition of the two wave at the focal point but were unable to fully clarify the reasons for the banded structure of the bubble field.

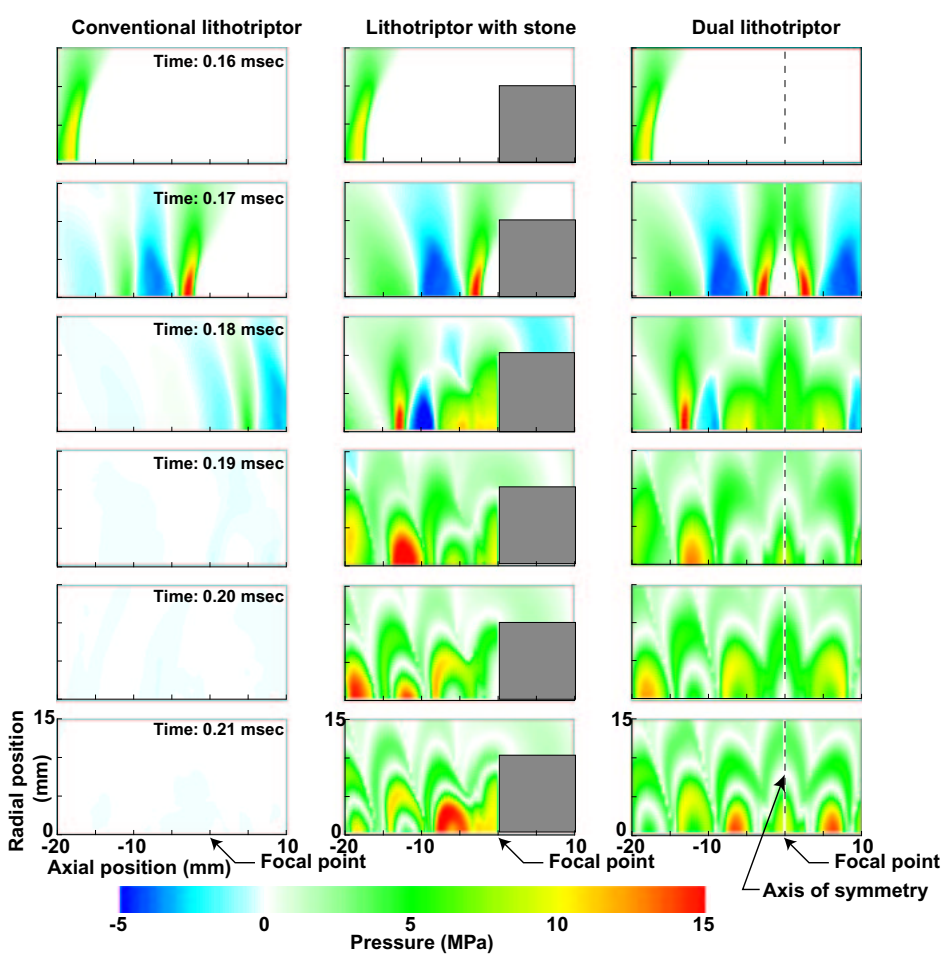

Figure 9. Comparison of pressure contours near $F_{2}$ for different lithotripter configurations

It is noted that a rigid stone of typical dimensions gives very

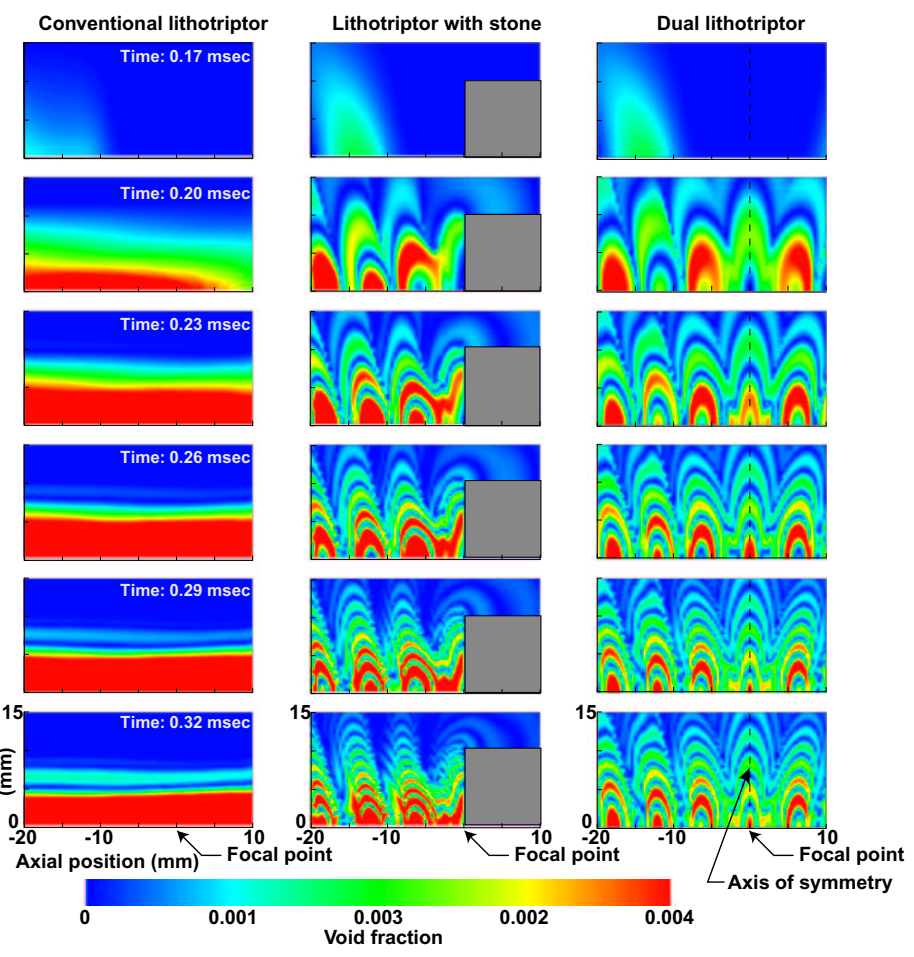

Figure 10. Comparison of the void fraction near $F_{2}$ for different lithotripter configurations

similar behavior to the dual-pulse case. The introduction of the stone in the calculations is still at a preliminary stage. A more detailed analysis is currently underway and corroboration of the results with some experimental observations will be forthcoming.

In the dual-pulse calculations, the expected size and duration increase measured in experiments was not observed. The bubbles grew to a maximum size of approximately $0.75 \mathrm{~mm}$ and lasted up to the order of $100 \mu \mathrm{s}$. However, as seen in figure 10, the bubble cloud exhibit a clear banded structure. Additional numerical tests performed with a $1 \mu \mathrm{m}$ initial bubble radius showed a very similar structure. These bubble formations are a direct consequence of the coupled interaction between the propagation of the ESWL pulse in the mixture and the bubble dynamics. However, at this stage, we cannot formulate an precise explanation for the nature of these structures and the parameters that determine their dimensions.

\section{CONCLUSION}

In this paper, we presented our numerical simulation of cavitation in lithotripsy for three cases: free field, artificial stone at focus and dual-pulse lithotripter. With the exception of bubble rebound after violent collapse, the free field calculation for 
an electro-hydraulic lithotripter compared very well with experimental observations.

As for the dual-pulse results, individual bubbles did not grow to the levels observed in experiments. Collectively, the cloud of bubbles exhibited a banded pattern which has been previously observed. Further analysis will be required in order to explain in greater detail the nature of the interaction between the mixture and bubble dynamics which generate these structures.

The calculations involving an artificial stone at the focal point of the lithotripter presented close similarities with the results from the dual-pulse configuration. Further investigations are needed before the present model can be used to study cavitation near the stone surface but even at this preliminary stage, we believe that this approach presents great potential as a numerical tool for the study of stone comminution in shock wave lithotripsy.

\section{Acknowledgments}

We would like to thank Christopher Brennen and Al Preston for their helpful suggestions and insight. We would also like to thank the PPG investigators, particularly Mike Bailey and Dahlia Sokolov for their comments as well as for sharing their experimental data. This work was supported by NIH under grant PO1 DK43881.

\section{REFERENCES}

Averkiou, M. \& R. Cleveland (1999). Modeling of an electrohydraulic lithotriptor with the KZK equation. Journal of the Acoustical Society of America 106(1), 102-112.

Bailey, M., D. Blackstock, R. Cleveland \& L. Crum (1999). Comparison of electrohydraulic lithotripters with rigid and pressure-release ellipsoidal reflectors. II. cavitation fields. Journal of the Acoustical Society of America 106, 1149-1160.

Biesheuvel, A. \& L. van Wijngaarden (1984). Two-phase flow equations for a dilute dispersion of gas bubbles in liquid. Journal of Fluid Mechanics 148, 301-318.

Cleveland, R., S. O.A., M. Bailey \& L. Crum (2000). A dual passibe cavitation detector for localized detection of lithotripsy-induced cavitation in vitro. Journal of the Acoustical Society of America 107(3), 1745-1758.

Coleman, A. \& J. Saunders (1993). A review of the physical properties and biological effects of the high amplitude acoustic fields used in extracorporeal lithotripsy. Ultrasonics 31(2), 75-89.

Colonius, T., S. Lele \& P. Moin (1993). Boundary conditions for direct computation of aerodynamic sound generation. AIAA Journal 31(9), 1574-1582.

Jiang, G.-S. \& C.-W. Shu (1996). Efficient implementation of weighted eno schemes. Journal of Computational
Physics 126, 202-228.

Kumar, S. \& C. Brennen (1993). Some nonlinear interactive effects in bubbly cavitating clouds. Journal of Fluid Mechanics 253, 565-591.

Liu, X.-D., S. Osher \& T. Chan (1994). Weighted essentially nonoscillatory schemes. Journal of Computational Physics 115, 200-212.

Lokhandwalla, M. \& B. Sturtevant (2001). Mechanical haemolysis in shock wave lithotripsy (SWL): I. analysis of cell deformation due to SWL flow-fields. Physics in Medicine and Biology 46, 1-25.

Matula, T., P. Hilmo, B. Storey \& A. Szeri (2002). Radial response of individual bubbles subjected to shock wave lithotripsy pulses in vitro. Physics of Fluids 14(3), 913921.

Mohseni, K. \& T. Colonius (2000). Numerical treatment of polar coordinate singularities. Journal of Computational Physics 157, 787-795.

Poinsot, T. \& S. Lele (1992). Boundary conditions for direct simulations of compressible viscous flows. Journal of Computational Physics 101(1), 104-129.

Preston, A., T. Colonius \& C. Brennen (2002). A reducedorder model of heat transfer effects on the dynamics of bubbles. In Proceedings of FEDSM'02 Cavitation and Multiphase Flow Forum, Montreal, Canada. ASME.

Sokolov, D., M. Bailey \& L. Crum (2001). Use of a dualpulse lithotripter to generate a localized and intensified cavitation field. Journal of the Acoustical Society of America 110, 1685-1695.

Sokolov, D., M. Bailey, F. Pulvermaker \& L. Crum (2000). Increased damage to stones without increased damage to cells with a dual-reflector lithotripter. In presented at the 2000 IEEE International Ultrasonics Symposium, San Juan, Puerto Rico.

Tanguay, M. \& T. Colonius (2001). Numerical simulation of bubbly cavitating flow in shock wave lithotripsy. In CAV2001 fourth international symposium on cavitation. California Institute of Technology.

Wang, Y.-C. (1999). Effects of nuclei size distribution on the dynamics of a spherical cloud of cavitaion bubbles. Journal of Fluids Engineering 121, 881-886.

Willis, L., A. Evan, B. Connors, P. Bloomgren \& J. Lingeman (1999). The impact of high-dose lithotripsy on renal structure and function. Contemporary Urology, 45-50.

Zhang, D. \& A. Properetti (1994a). Averaged equations for inviscid disperse two-phase flow. Journal of Fluid Mechanics 267, 185-219.

Zhang, D. \& A. Properetti (1994b). Ensemble phase-averaged equations for bubbly flow. Physics of Fluids 6(9), 29562970. 